\title{
Bone marrow stromal cells enhance the survival of chronic lymphocytic leukemia cells by regulating HES-1 gene expression and H3K27me3 demethylation
}

\author{
ZHENSHU XU* ${ }^{*}$ DONGLIAN XIONG* , JUSHUN ZHANG, JINGYAN ZHANG, \\ XIULI CHEN, ZHIZHE CHEN and RONG ZHAN \\ Fujian Institute of Hematology, Fujian Provincial Key Laboratory of Hematology, \\ Fujian Medical University Union Hospital, Fuzhou, Fujian 350001, P.R. China
}

Received September 21, 2015; Accepted March 9, 2017

DOI: $10.3892 / \mathrm{ol} .2017 .7450$

\begin{abstract}
The majority of patients with chronic lymphocytic leukemia (CLL) are not cured by traditional chemotherapy. One possibleexplanationforthisis that themicroenvironmentprotects CLL cells from both spontaneous- and cytotoxic-mediated apoptosis. The present study was designed to investigate the mechanisms accounting for these effects, since this information is crucial to understanding CLL physiopathology and identifying potential treatment targets. The CLL cell line L1210 and primary CLL cells were cultured under different conditions: With serum, cyclophosphamide (CTX), or with monolayers and conditioned medium (CM) from the stromal cell line HESS-5. Apoptosis, Hes family BHLH transcription factor 1 (HES-1) gene and protein expression, and histone H3K27me3 DNA demethylation were determined. Co-culture of L1210 cells with HESS-5 cells significantly inhibited serum deprivation- and CTX-induced apoptosis of leukemia cells, and resulted in a significant increase in short-term proliferation. Soluble factors in the CM from HESS-5 cells had a negligible effect. The HESS-5 cell-mediated inhibition of apoptosis of CLL cells was associated with increased HES-1 expression and hypomethylation of the H3K27me3 gene in the leukemia cells. These results indicate that stromal cells enhance the survival of CLL cells by regulating the HES-1 gene and protein expression, as well as H3K27me3 DNA demethylation, and suggest that specific interactions between stromal and
\end{abstract}

Correspondence to: Dr Zhenshu Xu or Professor Rong Zhan, Fujian Institute of Hematology, Fujian Provincial Key Laboratory of Hematology, Fujian Medical University Union Hospital, 29 Xinquan Road, Fuzhou, Fujian 350001, P.R. China

E-mail: zhenshuxu@yahoo.com

E-mail: deanzhanrong@qq.com

*Contributed equally

Key words: CLL, stromal cells, HES-1, H3K27me3, demethylation leukemia cells may enhance the resistance of leukemia cells to chemotherapy.

\section{Introduction}

Chronic lymphocytic leukemia (CLL) is the most common type of adult leukemia in the Western hemisphere (1). In 2016, 18 960 new cases of CLL were diagnosed in the United States (1). The treatment of CLL has markedly changed in the past years with the regulatory approval of idelalisib and ibrutinib. In addition, other antibodies are likely to become widely available in the next few years $(2,3)$. Over the past decade, the essential role of the tumor microenvironment in the survival and progression of CLL has become increasingly clear (4). In the bone marrow and secondary lymphatic tissues, CLL cells engage in complex, yet not completely defined, cellular and molecular interactions with stromal cells and the matrix, which collectively are referred to as the 'microenvironment' (5). These interactions affect CLL cell survival and proliferation, and confer drug resistance, which may be responsible for residual disease following conventional therapy (6). Understanding the association of neoplastic cells with the microenvironment (i.e., identifying which cells are required for lymphoma growth and which are involved in regulating lymphoma growth) will be crucial to developing therapies aimed at targeting the microenvironment $(4,6)$. Notably, the cross-talk between CLL cells and bone marrow stromal cells (BMSCs) is bidirectional, causing the activation of both CLL cells and BMSCs (7). BMSCs attract and affect CLL cells via G-protein-coupled chemokine receptors, including $\mathrm{C}-\mathrm{X}-\mathrm{C}$ chemokine receptor type 4, cluster of differentiation (CD) 79a and Notch homolog 1, translocation-associated (Drosophila) (Notch1), which are expressed at high levels on CLL cells $(8,9)$. Our group previously reported that Notch1 constitutively activates the nuclear factor $(\mathrm{NF})-\kappa \mathrm{B}$ signaling pathway through activating its downstream gene, Hes family BHLH transcription factor 1 (HES-1), in CLL (10). However, the mechanism by which stromal cells affect Notch1-driven oncogenic target gene activation by histone modification is largely unknown. The present study demonstrates that stromal cells enhance the survival of CLL cells by regulating HES-1 gene and protein expression, as 
well as histone H3K27me3 DNA demethylation. In the present study, several experiments were designed to determine if a BMSC line, HESS-5, improves the survival and proliferation of CLL cells in vitro. The ability of HESS-5 cells to protect CLL cells from serum deprivation-induced apoptosis and to modify apoptosis responses to the chemotherapy agent cyclophosphamide (CTX) were evaluated. In addition, the present study examined whether the expression of HES-1, one of the key regulators of apoptosis, as well as $\mathrm{H} 3 \mathrm{~K} 27 \mathrm{me} 3$, the main histone involved in the gene transcription of Notch1 (11), are affected in CLL cells by co-culture with HESS-5 monolayers.

\section{Materials and methods}

Cells. The hematopoietic-supportive murine stromal cell line HESS-5, murine CLL cell line L1210 (The Cell Bank of Type Culture Collection of Chinese Academy of Sciences, Shanghai, China), and primary CLL cells from patients with CLL were used in the present study. The choice of L1210 was due to ease of accessibility and culturing compared with other human CLL cell lines in our lab. Samples $(10 \mathrm{ml})$ of human bone marrow were obtained from a newly diagnosed patient with CLL upon obtaining informed consent in Fujian Medical University Union Hospital (Fuzhou, China) between 1st January 2012 and 1st January 2014. The present study was approved by the Ethics Committee of Fujian Medical University Union Hospital (document no. 139). The L1210 and primary CLL cells were cultured in 24-well, flat-bottom plates, with or without a confluent HESS-5 stromal cell layer in RPMI-1640 with $20 \%$ fetal bovine serum, $1 \%$ penicillin and streptomycin [American Type Culture Collection (ATCC), Manassas, VA, USA]. All cells were cultured at $37^{\circ} \mathrm{C}$ with $5 \%$ $\mathrm{CO}_{2}$. In certain experiments, cells were grown in serum-free conditions for $24 \mathrm{~h}$, with or without the presence of 0.5 or $1.0 \mu \mathrm{M}$ CTX (GE Healthcare Life Sciences, Uppsala, Sweden).

L1210 cell growth on fixed stromal cells. To interrupt the metabolic activity of stromal cells while leaving surface proteins intact, confluent HESS-5 cell layers were treated with glutaraldehyde as previously described (12). L1210 cells were then incubated on the fixed stromal cell layers for $48 \mathrm{~h}$ in the presence of CTX $(0.5$ or $1.0 \mu \mathrm{M})$. L1210 cells were collected through vigorous pipetting, and the viability of all samples was evaluated in triplicate by Trypan Blue exclusion assay as previously described (12).

Evaluation of leukemic cell viability. L1210 cells were cultured under different conditions: With or without serum, CTX $(0.5$ or $1.0 \mu \mathrm{M})$, monolayers or $50 \%$ stromal conditioned medium (CM) from HESS-5 cells. Viability was evaluated by Trypan Blue exclusion assay in triplicate samples daily. After $48 \mathrm{~h}$ of the above treatments, leukemic cells were transferred to 96-well flat-bottom plates containing fresh RPMI-1640 medium with $10 \%$ fetal calf serum (FCS; ATCC). Viability was evaluated using an MTT assay as previously described following an additional $48 \mathrm{~h}$ (12).

Evaluation of leukemic cell apoptosis. CLL cells or L1210 were cultured under different conditions: With or without CTX, monolayers or $50 \%$ stromal CM of HESS-5 cells. CLL cells or L1210 were removed from adherent stromal cells by vigorous pipetting, and then evaluated using flow cytometry as previously described (12) with an Alexa Fluor488 AnnexinV apoptosis kit (Gibco; Thermo Fisher Scientific, Inc., Waltham, MA, USA) according to the manufacturer's protocol. For the terminal deoxynucleotidyl transferase-mediated 2'-deoxyuridine 5'-triphosphate (dUTP) nick end labeling (TUNEL) assay, fluorescein-conjugated dUTP incorporated into nucleotide polymers was detected and quantified using flow cytometry. In the present study, the In Situ Cell Death Detection kit, Fluorescein (Roche Diagnostics GmbH, Mannheim, Germany) was used according to the manufacturer's protocol. Briefly, cells $\left(2 \times 10^{6}\right.$ cells/well) were cultured on cover glasses in 6-well plates. Following treatment, cells were washed with PBS, fixed with $4 \%$ paraformaldehyde solution for $1 \mathrm{~h}$ at $20-25^{\circ} \mathrm{C}$ and permeabilized through incubation for $2 \mathrm{~min}$ on ice. Following washing with PBS cells were incubated with the TUNEL reaction mixture for $60 \mathrm{~min}$ at $37^{\circ} \mathrm{C}$ in the dark. Fluorescence of the stained cells was quantified using flow cytometry.

$R N A$ isolation and reverse transcription-polymerase chain reaction $(R T-P C R)$. After $24 \mathrm{~h}$ of $\mathrm{CTX}$ treatment, primary CLL cells were transferred onto a confluent HESS-5 cell layer and cultured for $\leq 48 \mathrm{~h}$. Leukemic cells were collected by vigorous pipetting. Their RNA was isolated according to the single-step acid guanidinium thiocyanate-phenol-chloroform (Sigma-Aldrich; Merck KGaA, Darmstadt, Germany) method (13). The RNA quality and quantity were determined following $1.0 \%$ agarose gel electrophoresis and staining with $1 \mu \mathrm{g} / \mathrm{ml}$ ethidium bromide. Oligo-(dT) 10 primers (Invitrogen; Thermo Fisher Scientific, Inc.) were used as for complementary DNA (cDNA) synthesis. Total RNA template was used per each $10 \mu \mathrm{l}$ of RT reaction in the presence of AMV Reverse Transcriptase (Roche Diagnostics $\mathrm{GmbH}$ ) at $42^{\circ} \mathrm{C}$ for $1 \mathrm{~h}$. PCR results were confirmed using $1 \%$ agarose gel electrophoresis then stored at $-20^{\circ} \mathrm{C}$. PCR amplification reaction mixtures (50 $\mu \mathrm{l})$ contained DNA polymerase, dNTPs, cDNA $(2 \mu \mathrm{l})$, and HES-1, c-MYC forward/reverse primers (70 pmol/l) and $\beta_{2}$-microglobulin primers (70 pmol/l; used as control), which were synthesized by Shanghai Sangong Pharmaceutical Co., Ltd. (Shanghai, China). Thermal cycler conditions included holding the reactions at $50^{\circ} \mathrm{C}$ for $2 \mathrm{~min}$ and at $95^{\circ} \mathrm{C}$ for $10 \mathrm{~min}$, followed by amplification for 40 cycles using $95^{\circ} \mathrm{C}$ for $15 \mathrm{sec}$ and $60^{\circ} \mathrm{C}$ for $1 \mathrm{~min}$. The PCR samples were electrophoresed on $1.0 \%$ agarose gels and visualized with $1 \mu \mathrm{g} / \mathrm{ml}$ ethidium bromide. The gel images were digitally captured and analyzed with AlphaEaseFC software (version 5.0; ProteinSimple; Bio-Techne, Minneapolis, MN, USA).

Western blot analysis of HES-1 expression. After $24 \mathrm{~h}$ of CTX $(0.5$ or $1.0 \mu \mathrm{M})$ treatment, primary CLL cells were transferred onto the confluent HESS-5 cell layer for $\leq 48 \mathrm{~h}$. Leukemic cells were collected by vigorous pipetting following either 24 or $48 \mathrm{~h}$ of co-culture, and then lysed in $10 \mathrm{mM}$ 4-(2-hydroxyethyl)-1-piperazineethanesulfonic acid ( $\mathrm{pH} 7.6$ ), $250 \mathrm{mM} \mathrm{NaCl}, 5 \mathrm{mM}$ EDTA and $0.5 \%$ Nonidet P-40. The lysates were fractionated by $12 \%$ SDS-PAGE and transferred to nitrocellulose membranes. The membranes were then blocked in 5\% non-fat milk at room temperature for $1 \mathrm{~h}$ and then incubated with mouse anti-human HES-1 antibody (cat 
A

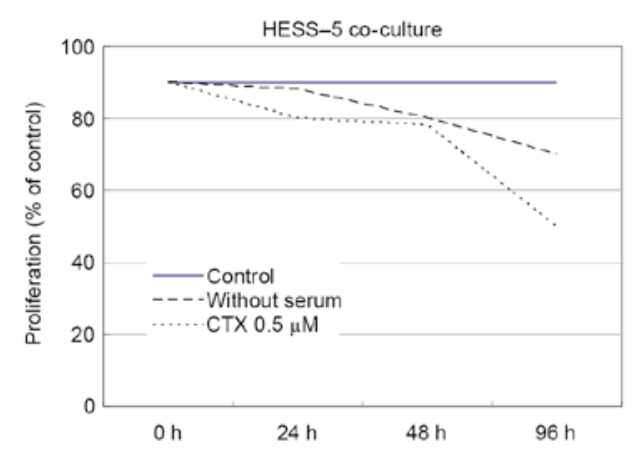

C

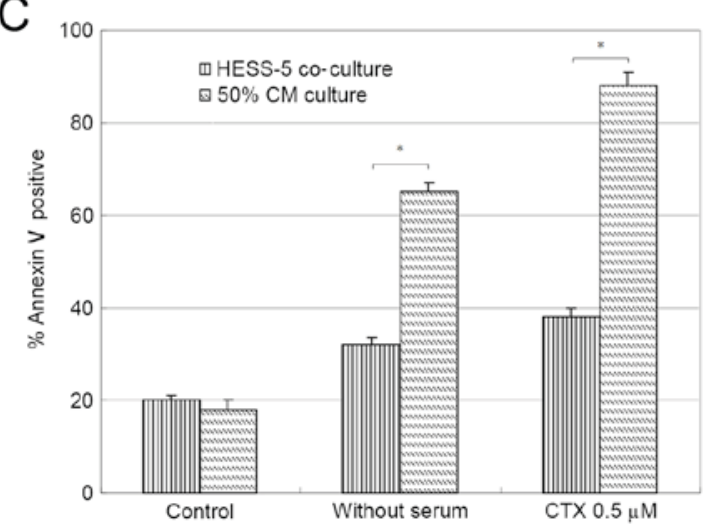

B

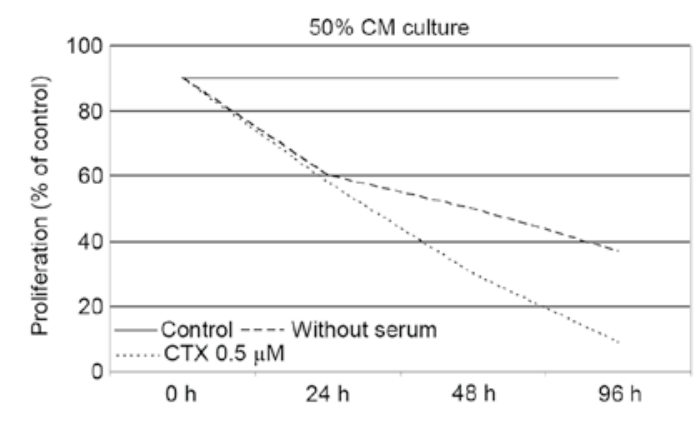

D

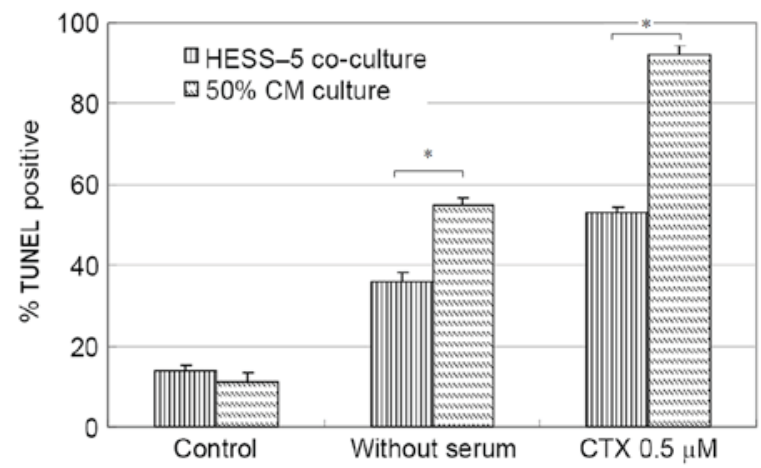

Figure 1. HESS-5 cells protect the L1210 chronic lymphocytic leukemia cell line from apoptosis and growth inhibition induced by serum deprivation or CTX. L1210 cells were cultured under different conditions: With or without serum, CTX, stromal cell (A) HESS-5 monolayers or (B) 50\% stromal CM from HESS-5 cells. Cell growth (percentage of control) was assayed in triplicate wells. The results represent the average of $\geq 3$ independent experiments. (C) Annexin $\mathrm{V}$ staining of L1210 cells treated for $48 \mathrm{~h}$ as indicated. ("P<0.01, HESS- 5 coculture vs. CM culture) (D) TUNEL assay of L1210 cells treated for $48 \mathrm{~h}$ as indicated. ( $\mathrm{P}<0.01$, HESS -5 coculture vs. CM culture) The results are the average of three independent experiments. CTX, cyclophosphamide; CM, conditioned medium; TUNEL, terminal deoxynucleotidyl transferase-mediated dUTP nick end labeling.

A
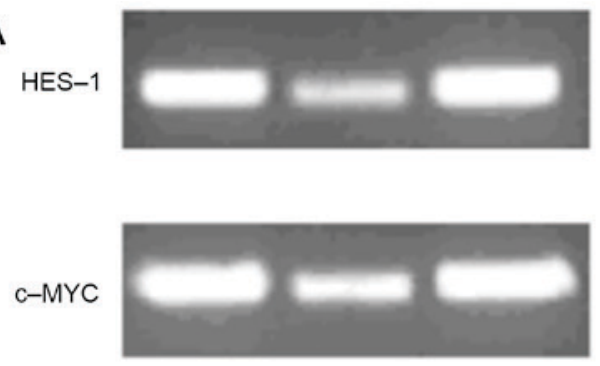

$\beta 2-M G$
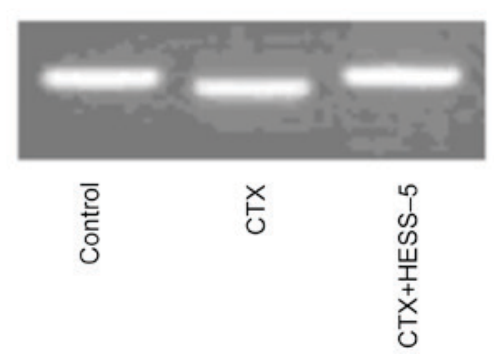

B

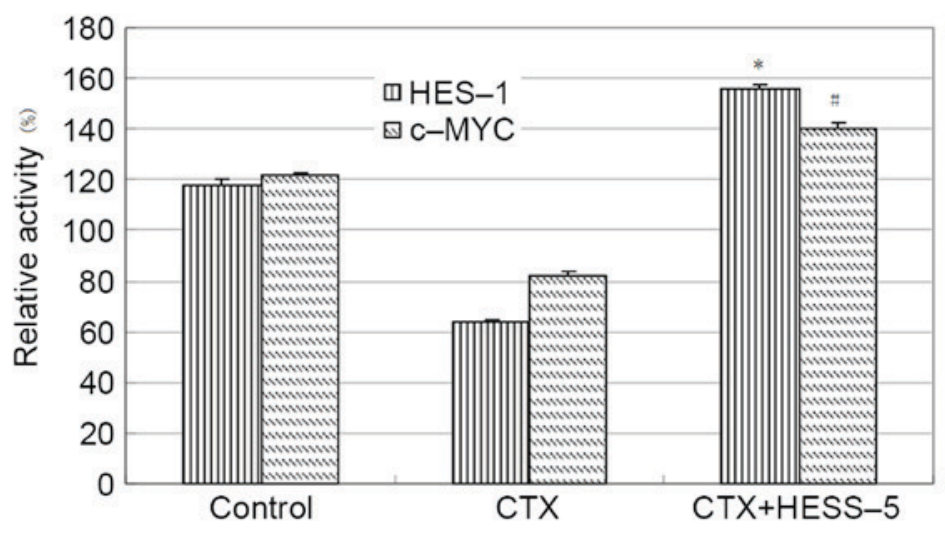

Figure 2. Messenger RNA expression of HES-1 and c-MYC in CTX-treated CLL cells cultured alone or in the presence of stromal HESS-5 cells. (A) Amplification and detection were performed by semi-quantitative reverse transcription-polymerase chain reaction. Primary CLL cells exhibited inhibition of HES-1 and c-MYC expression following CTX treatment, which was recovered in the presence of the stromal HESS-5 cell monolayer. A representative graph of three independent experiments is presented. (B) HES-1 and c-MYC expression levels were determined following different treatments, and normalized to $\beta_{2}-\mathrm{MG}(\%)$. The results revealed higher HES-1 and c-MYC expression in primary CLL cells co-cultured with HESS-5 cells compared with that observed in primary CLL cells cultured in the absence of HESS- 5 cells following CTX exposure ( ${ }^{*}, \mathrm{P}<0.05$ vs. the CTX group). Data are shown as the mean + standard error of the mean, and are representative of three independent experiments. HES-1, Hes family BHLH transcription factor 1; CTX, cyclophosphamide; $B_{2}$-MG, $\beta_{2}$-microglobulin; CLL, chronic lymphocytic leukemia. 
no.RS-2972R; 1:3,000 dilution; Santa Cruz Biotechnology,Inc., Dallas, TX, USA) and a mouse monoclonal anti- $\beta$-actin antibody (cat no. A5316; control; 1:3,000 dilution; Sigma-Aldrich; Merck $\mathrm{KGaA}$ ) overnight at $4^{\circ} \mathrm{C}$, and a secondary horseradish peroxidase-conjugated anti-mouse antibody (cat no. BL0824; Beijing Zhongshan Jinqiao Biotechnology Co., Ltd., Beijing, China) for $1 \mathrm{~h}$ at room temperature. Detection was performed with Immobilon Western Detection reagents (Sigma-Aldrich; Merck KGaA).

Methylation-specific PCR (MSP) analysis of the H3K27me3 gene. Primary CLL cells were seeded onto a confluent HESS- 5 cell layer for $\leq 48 \mathrm{~h}$. Genomic DNA was extracted from primary CLL cells following the standard procedure of DNA extraction kit (GE Healthcare Life Sciences). The genomic DNA was quantified by Qubit Fluorimeter (Thermo Fisher Scientific, Inc.) and then stored at $-20^{\circ} \mathrm{C}$. Approximately 5,000 ng DNA fragments were used for bisulfite conversion using the Qiagen EpiTect system (Qiagen, Inc., Valencia, CA, USA) according to the manufacturer's protocol, and $100 \mathrm{ng}$ of this modified DNA was used as the template for MSP analysis as previously described (14). Thermal cycler conditions included holding the reactions at $50^{\circ} \mathrm{C}$ for $2 \mathrm{~min}$ and at $95^{\circ} \mathrm{C}$ for $10 \mathrm{~min}$ per cycle, for a total of 33 cycles. The PCR samples were electrophoresed on $1.0 \%$ agarose gels and then stained with $1 \mu \mathrm{g} / \mathrm{ml}$ ethidium bromide. The gel images were digitally captured and analyzed with AlphaEaseFC software (version 5.0). U266 cells expressing methylated $\mathrm{H} 3 \mathrm{~K} 27 \mathrm{me} 3$ were used as a positive control (15).

Statistical analysis. Data are presented as the mean + the standard error of the mean. Statistical significance was determined by a Student's two-tailed t-test using SPSS version 11.0 (SPSS Inc., Chicago, IL, USA). $\mathrm{P}<0.05$ was considered to indicate a statistically significant difference.

\section{Results}

Stromal cells enhance CLL cell viability in serum-deprivation conditions and during chemotherapy exposure. To determine whether co-culture with a stromal cell monolayer affected leukemic cell survival during chemotherapy drug exposure and serum-deprivation, L1210 cells were grown on HESS-5 monolayer and compared with L1210 cells maintained in culture medium alone. In all cases, stromal cell co-culture resulted in increased L1210 cell viability during treatment (data not shown). The viability of CLL cells cultured on stromal cell layers increased by $\sim 3$-fold when compared with that of cells cultured in medium alone. Upon treatment with $0.5 \mu \mathrm{M}$ CTX for $48 \mathrm{~h}$, the CLL cell number decreased by $30 \%$, but stromal cell co-culture significantly rescued the viability of these CLL cells. After $96 \mathrm{~h}$ of serum-deprivation, the number of CLL cells cultured in medium alone was reduced by $40 \%$ compared with $70 \%$ when serum-deprived cells were co-cultured with HESS-5 cells (Fig. 1A).

Following serum-free culture or CTX exposure, CLL cells were co-cultured with stromal cells for $48 \mathrm{~h}$, prior to being collected and plated on 96-well flat-bottom plates with fresh medium (10\% FCS). Soluble factors derived from stromal cells $(50 \% \mathrm{CM})$ did not alter the response of

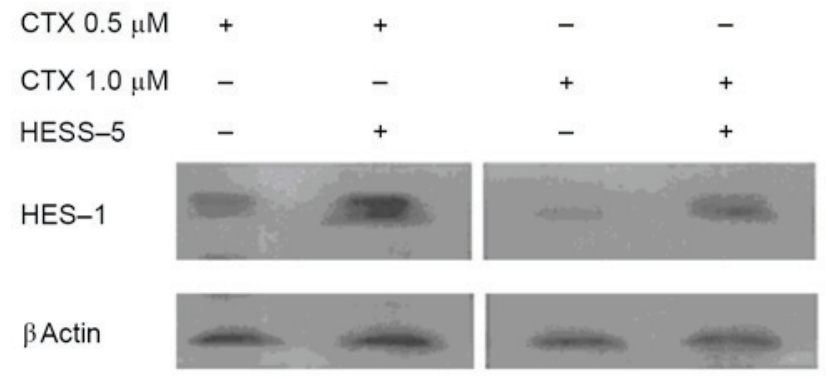

Figure 3. HESS-5 cells restore HES-1 protein expression in primary CLL cells. Western blot analysis of HES-1 protein expression in primary CLL cells treated with CTX $(0.5$ or $1.0 \mu \mathrm{M})$ for $24 \mathrm{~h}$, followed by additional $48 \mathrm{~h}$ of co-culture with a HESS- 5 cell monolayer. A representative image of three independent experiments is shown. HES-1, Hes family BHLH transcription factor 1; CTX, cyclophosphamide; CLL, chronic lymphocytic leukemia.
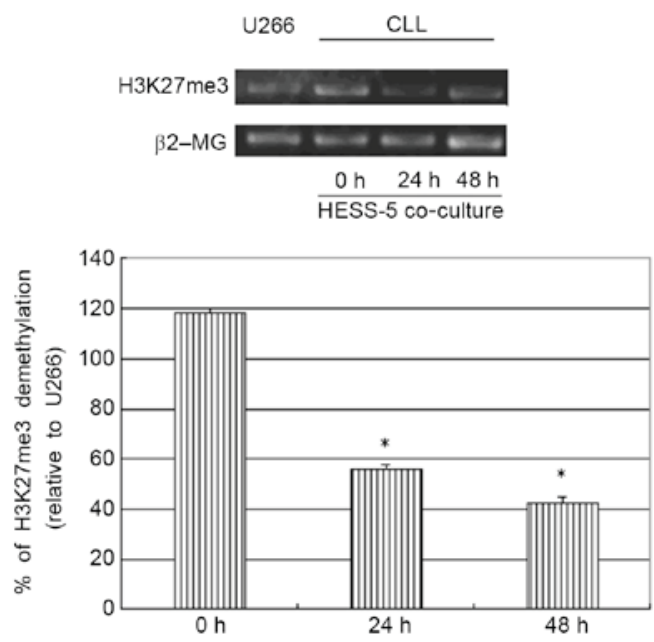

Figure 4. MSP analysis of histone $\mathrm{H} 3 \mathrm{~K} 27 \mathrm{me} 3$ methylation status Amplification and detection were performed by MSP. The H3K27me3 gene was shown to be methylated in primary CLL cells. However, co-culture with HESS-5 cells led to demethylation of this gene in CLL cells. The data shown are representative of three independent experiments ( $\mathrm{P}<0.05$ vs. U266 cells). MSP, methylation-specific PCR; $\beta_{2}$-MG, $\beta_{2}$-microglobulin; CLL, chronic lymphocytic leukemia.

leukemic cells to serum withdrawal or chemotherapy treatment (Fig. 1B).

Stromal cells inhibit leukemic cell apoptosis induced by CTX. To determine whether CTX induced apoptosis, L1210 cells were evaluated by flow cytometry, expression following chemotherapy drug exposure in the presence or absence of HESS-5 monolayer. The number of apoptotic cells increased from $20 \%$ in the control group to $90 \%$ in the experimental group after $48 \mathrm{~h}$ of CTX treatment. However, co-culture with stromal cells following CTX exposure resulted in significantly reduced numbers of apoptotic leukemic cells $(40 \%$; $\mathrm{P}<0.01$; Fig. 1C). The same results were also attained by TUNEL assay (Fig. 1D).

BMSCs enhance the expression of HES-1 and c-MYC in CLL cells following treatment with CTX. To further explore the mechanism of stromal cell protection of leukemic cells from apoptosis, HES-1 and c-MYC messenger RNA expression, as 
well as HES-1 protein expression, were measured in primary CLL cells treated with CTX in the presence or absence of a HESS-5 monolayer. HES-1 and c-MYC gene expression was higher in CLL cells supported by stromal cells than in CTX-treated CLL cells alone (Fig. 2A and B). Western blot analysis revealed that HES-1 protein expression was increased in primary CLL cells treated with two concentrations of CTX $(0.5$ and $1.0 \mu \mathrm{M})$ for $24 \mathrm{~h}$ and then cultured for additional $48 \mathrm{~h}$ with the HESS-5 cell monolayer (Fig. 3).

Co-culture of primary CLL cells with stromal cells results in demethylation of $H 3 K 27 m e 3$. To further explore the possible mechanism of stromal cell protection of leukemic cells from apoptosis, H3K27me3 gene expression was measured in primary CLL cells treated with CTX in co-culture with a HESS-5 stromal cell layer. The H3K27me3 gene has been shown to be methylated in primary CLL cells (16). However, in the present study, co-culture with HESS-5 cells led to a significant demethylation of this gene in primary CLL cells ( $\mathrm{P}<0.05$; Fig. 4).

\section{Discussion}

Growth and differentiation in vivo of hematopoietic cells, including normal and leukemic cells, require direct contact of hematopoietic cells with stromal cells (4). CLL cells display enhanced survival in vivo, but they die spontaneously and are difficult to maintain alive in vitro $(5,7)$. It has been reported that BMSCs synthesize several cytokines, including colony stimulating factors, interleukin (IL)-6, IL-7, IL-10, transforming growth factor- $\beta$ and stem cell factor, which constitute a complex regulatory network together with extracellular matrix proteins (17). Contact with the bone marrow stroma induces the proliferation and survival of acute myeloid leukemia, acute lymphoblastic leukemia and CLL cells in culture $(17,18)$. HESS-5 cells, a murine stromal cell line capable of supporting early human progenitors in vitro (19), was used in the present study as a model to evaluate hematopoietic-stromal cell interactions.

The present study observed that stromal cell CM conferred negligible protection for leukemic cells during serum deprivation or CTX-induced apoptosis. Therefore, the role of proteins on the stromal cell surface was investigated using glutaraldehyde-fixed stromal cells. Previous reports have shown that this fixation process disrupts the metabolism of adherent 3T3 cells, thus allowing to investigate the role of signaling through ligand-receptor interactions in the absence of cytokine production (20). It has also been suggested that signals between stromal cells and hematopoietic progenitors can be bidirectional, with the adhesion of hematopoietic cells to stromal cells altering stromal cell function (21). In the present study, fixed stromal cells protected leukemic cells from CTX-induced cell death, similarly to viable stromal cells. These observations support the conclusion that the signals protecting leukemic cell survival primarily result from cell-surface interactions, but not from soluble factors in stromal CM.

The present study also demonstrated that HESS-5 cells mediated the inhibition of apoptosis that was associated with increased HES-1 and c-MYC expression. Notch1 is known to be involved in the pathogenesis of leukemia. Unlike normal
B cells, CLL cells express both Notch1 and 2, and their ligands (10). Co-expression of the Notch receptors and their ligands results in the constitutive activation of the Notch target molecule HES-1, which is a potential biomarker in leukemia cells $(22,23)$. In CLL, upon Notch1 activation, the cleaved intracellular portion of the Notch1 receptor translocates into the nucleus, where it recruits a transcription complex to modify the expression of several target genes, including HES-1, c-MYC and NF- $\kappa$ B (10). Notch1 mutations in CLL have been reported to cause disruptions to HES-1 expression, resulting in the stabilization of active intracellular Notch1 and deregulated Notch signaling (24). In fact, Notch1 mutations are significantly more common in CLL harboring +12 trisomy with intermediate-risk disease compared with low-risk CLL $(25,26)$. Although stromal cells were demonstrated to stimulate the expression of HES-1 and c-MYC in CLL cells in the present study, further studies are warranted to evaluate whether stromal cells can activate mutated Notch1 in a similar manner.

CLL hypermethylation has been reported to frequently affect DNA repeats (27). In general, hypermethylation frequently targets genes already silenced in non-tumor cells by repressive histone modifications such as H3K27me3 (28). Thus, although certain tumor-suppressor genes become de novo methylated and silenced in cancer, hypermethylation affects mostly genes already silenced in normal cells $(28,29)$. The H3K27me3 methylation mark is associated with chromatin condensation and transcriptional repression (16). The results of the present study confirmed that stromal cells in direct contact with CLL cells lead to the demethylation of H3K27me3, which is associated with transcriptional activation of the target gene HES-1.

As the present study was performed using the mouse CLL line, further studies are required to validate the present results in human CLL cell lines. In summary, the present study has shown that cell-cell contact with BMSCs protects CLL cells from apoptosis by upregulating HES-1 and c-MYC expression, which is associated with $\mathrm{H} 3 \mathrm{~K} 27 \mathrm{me} 3$ demethylation. Further studies will be necessary to identify other factors and mechanisms involved in the interaction between stromal and leukemic cells.

\section{Acknowledgements}

The present study was supported by the Fujian Natural Science Fund (grant no. 2016J01458) and the New Century Talent in Fujian (grant no. JA10128) (both received by Zhenshu Xu), sponsored by National and Fujian Provincial Key Clinical Specialty Discipline Construction Program, P.R.C and Construction Project of Fujian Medical Center of Hematology (Min201704).

\section{References}

1. Siegel RL, Miller KD and Jemal A: Cancer statistics, 2016. CA Cancer J Clin 66: 7-30, 2016.

2. Suresh T, Lee L, Joshi J and Barta S: New antibody approaches to lymphoma therapy. J Hematol Oncol 7: 58, 2014.

3. Wu J, Fu J, Zhang M and Liu D: Blinatumomab: A bispecific $T$ cell engager (BiTE) antibody against CD19/CD3 for refractory acute lymphoid leukemia. J Hematol Oncol 8: 104, 2015. 
4. Nwabo Kamdje AH and Krampera M: Notch signaling in acute lymphoblastic leukemia: Any role for stromal microenvironment. Blood 118: 6506-6514, 2011.

5. Burger JA, Ghia P, Rosenwald A and Caligaris-Cappio F: The microenvironment in mature B-cell malignancies: A target for new treatment strategies. Blood 114: 3367-3375, 2009.

6. Sun Z, Wang S and Zhao RC: The roles of mesenchymal stem cells in tumor inflammatory microenvironment. J Hematol Oncol 7: 14, 2014

7. Ding W, Nowakowski GS, Knox TR, Boysen JC, Maas ML, Schwager SM, Wu W, Wellik LE, Dietz AB, Ghosh AK, et al: Bi-directional activation between mesenchymal stem cells and CLL B-cells: Implication for CLL disease progression. Br J Haematol 147: 471-483, 2009.

8. Vlad A, Deglesne PA, Letestu R, Saint-Georges S, Chevallier N, Baran-Marszak F, Varin-Blank N, Ajchenbaum-Cymbalista F and Ledoux D: Down-regulation of CXCR4 and CD62L in chronic lymphocytic leukemia cells is triggered by B-cell receptor ligation and associated with progressive disease. Cancer Res 69: 6387-6395, 2009.

9. Wang L, Lawrence MS, Wan Y, Stojanov P, Sougnez C, Stevenson K, Werner L, Sivachenko A, DeLuca DS, Zhang L, et al: SF3B1 and other novel cancer genes in chronic lymphocytic leukemia. N Engl J Med 365: 2497-2506, 2011.

10. Xu ZS, Zhang JS, Zhang JY, Wu SQ, Xiong DL, Chen HJ, Chen ZZ and Zhan R: Constitutive activation of NF-kB signaling by NOTCH1 mutation in chronic lymphocytic leukemia. Onco Rep 33: 1609-1614, 2015.

11. Ross J, Mavoungou L, Bresenick EH and Milot E: GATA-1 utilizes lkaros and polycomb repressive complex 2 to suppress Hes-1 and to promote erythropoiesis. Mol Cell Biol 32: 3624-3638, 2012.

12. Hasslen SR, Burns AR, Simon SL,Smith CW,Starr K, Barclay AN, Michie SA, Nelson RD and Erlandsen SL: Preservation of spatia organization and antigenicity of leukocyte surface molecules by aldehyde fixation: Flow cytometry and high-resolution FESEM studies of CD62L, CD11b and Thy-1. J Histochem Cytochem 44: $1115-1122,1996$.

13. Chomozynski P and Sacchi N: Single step method of RNA isolation by acid guanidinium thiocyanate phenol chloroform extraction. Anal Biochem 162: 156-159, 1987.

14. Cai MY, Hou JH, Rao HL, Luo RZ, Li M, Pei XQ, Lin MC, Guan XY, Kung HF, Zeng YX and Xie D: High expression of $\mathrm{H} 3 \mathrm{~K} 27 \mathrm{me} 3$ in human hepatocellular carcinomas correlates closely with vascular invasion and predicts worse worse prognosis in patients. Mol Med 17: 12-20, 2011.

15. Kikuchi J, Koyama D, Wada T, Izumi T, Hofgaard PO, Bogen B and Furukawa Y: Phosphorylation-mediated EZH2 inactivation promotes drug resistance in multiple myeloma. J Clin Invest 125: 4375-4390, 2015

16. Amin S, Walsh M, Wilson C, Parker AE, Oscier D, Willmore E, Mann D and Mann J: Cross-talk between DNA methylation and active histone modifications regulates aberrant expression of ZAP70 in CLL. J Cell Mol Med 16: 2074-2084, 2012.

17. Moshaver B, van der Pol MA, Westra AH, Ossenkoppele GJ, Zweegman S and Schuurhuis GJ: Chemotherapeutic treatment of bone marrow stromal cells strongly affects their protective effect acute myeloid leukemia cell survival. Leuk Lymphoma 49: 134-148, 2008
18. Lagneaux L, Delforge A, Bron D, De Bruyn C and Stryckmans P: Chronic lymphocytic leukemic B cells are rescued from apoptosis by contact with normal bone marrow stromal cells. Blood 91: 2387-2396, 1998

19. Shimakura Y, Kawada H, Ando K, Sato T, Nakamura Y, Tsuji T, Kato S and Hotta T: Murine stromal cell line HESS-5 maintains reconstituting ability of ex vivo-generated hematopoietic stem cells from human bone marrow and cytokine-mobilized peripheral blood. Stem Cells 18: 183-189, 2000.

20. Roberts RA, Spooncer E, Parkinson EK, Lord BI, Allen TD and Dexter TM: Metabolically inactive 3T3 cells can substitute for marrow stromal cells to promote the proliferation and development of multipotent hematopoietic stem cells. J Cell Physiol 132: 203-214, 1987.

21. Jiang H, Sugimoto K, Sawada H, Takashita E, Tohma M, Gonda $\mathrm{H}$ and Mori KJ: Mutual education between hematopoietic cells and bone marrow stromal cells through direct cell-to-cell contact: Factors that determine the growth of bone marrow stroma-dependent leukemic (HB-1) cells. Blood 92: 834-841, 1998.

22. Smith AD, Roda D and Yap TA: Strategies for modern biomarker and drug development in oncology. J Hematol Oncol 7: 70, 2014.

23. Ma S, Shi Y, Pang Y, Dong F, Cheng H, Hao S, Xu J, Zhu X, Yuan W, Cheng T and Zheng G: Notch1-induced T cell leukemia can be potentiated by microenvironmental cues in the spleen. J Hematol Oncol 7: 71, 2014.

24. Fabbri G, Rasi S, Rossi D, Trifonov V, Khiabanian H, Ma J, Grunn A, Fangazio M, Capello D, Monti S, et al: Analysis of the chronic lymphocytic leukemia coding genome: Role of NOTCH1 mutational activation. J Exp Med 208: 1389-1401, 2011.

25. Balatti V, Bottoni A, Palamarchuk A, Alder H, Rassenti LZ, Kipps TJ, Pekarsky Y and Croce CM: NOTCH1 mutations in CLL associated with trisomy 12. Blood 119: 329-331, 2012.

26. Xu Z, Zhang J, Wu S, Zheng Z, Chen Z and Zhan R: Younger patients with chronic lymphocytic leukemia benefit from rituximab treatment: A single center study in China. Oncol Lett 5: 1266-1272, 2013.

27. Fabris S, Bollati V, Agnelli L, Morabito F, Motta V, Cutrona G, Matis S, Grazia Recchia A, Gigliotti V, Gentile M, et al: Biological and clinical relevance of quantitative global methylation of repetitive DNA sequences in chronic lymphocytic leukemia. Epigenetics 6: 188-194, 2011.

28. Martín-Subero JI, Kreuz M, Bibikova M, Bentink S, Ammerpohl O, Wickham-Garcia E, Rosolowski M, Richter J, Lopez-Serra L, Ballestar E, et al: New insights into the biology and origin of mature aggressive B-cell lymphomas by combined epigenomic, genomic, and transcriptional profiling. Blood 113: 2488-2497, 2009.

29. Lu Q, Lin X, Feng J, Zhao X, Gallagher R, Lee YM, Chiao JW and Liu DL: Phenylhexyl isothiocyanate has dual function as histone deacetylase inhibitor and hypomethylating agent and can inhibit myeloma cell growth by targeting critical pathways. J Hematol Oncol 1: 6, 2008. 